\title{
SCHNitTMUSTER DER PRÄSENTATION POPUläreR MUSIK: ÜBERLEGUNGEN ZUR ANALYSE AKTUELLER POPMUSIK AM BEISPIEL MAXİMO PARKS
}

\author{
André Doehring
}

\section{Einleitung}

Das Erkenntnisinteresse systematischer Musikwissenschaft liegt nach HansPeter Reinecke (1971: 10) darin, »bestimmte Sachverhalte oder Tatsachen der Musik bzw. Feststellungen über Musik als in regelmäßigen Verknüpfungsverhältnissen stehend zu erkennen und zu beschreiben«. Die von Reinecke hinzugefügte Aufforderung, diese Beziehungen »nach Möglichkeit « zu erklären und zu begründen, stellt die musikwissenschaftliche Auseinandersetzung mit aktueller populärer Musik, die vorwiegend dem Aufgabenbereich der systematischen Disziplinen zugeschlagen wird, vor eine gewaltige Aufgabe. Denn einerseits existiert nur wenig gegenwartssensitive Forschung, an deren Erkenntnisse anzuknüpfen wäre, andererseits ist man sich im Fach unsicher, welche Methoden geeignet scheinen, um sich dem aktuellen popmusikalischen Diskurs über Sachverhalte oder Tatsachen beziehungsweise Feststellungen über Musik zu nähern.

Im Jahr 2005 war dieser Diskurs davon bestimmt, dass mancher Zeitgenosse, zumal der im Medienbetrieb tätige, das Gefühl eines musikalischen déja-vu resp. déja-entendu hatte, wie sich aus diversen Äußerungen im Internet, in Musikmagazinen, Feuilletons und Resten des Musikfernsehens über dort vorgestellte Interpreten und Bands schließen lässt. Zwei musikalische Bereiche wurden 2005 besonders hervorgehoben: zum einen der so genannte »Deutschrock« von Bands wie Wir sind Helden, Juli oder Silbermond, ${ }^{1}$ zum anderen eine als "New Wave of New Wave " (Gross 2005: 64) titulierte Bewegung, die aufgrund bestimmter Merkmale als relativ homo-

1 Einige Muster benennt lesenswert Dietmar Dath (2005). 
gene Gruppe identifiziert wurde. ${ }^{2}$ Zu dieser Gruppe gehören vor allem die Bands Bloc Party, Kaiser Chiefs, The Bravery, The Killers, Editors, The Cribs, The Rakes, Art Brut, The Tears, The Others, Hard-Fi, The Futureheads und Maxïmo Park. Ihre wesentlichen Gemeinsamkeiten, die hier als Muster '05 interpretiert werden, lassen sich wie folgt systematisieren: Zum ersten veröffentlichten alle der genannten Bands in den Jahren 2004 und 2005 ihr Debütalbum. Zweitens kommen fast alle Bands aus Großbritannien, nur zwei stammen aus den Vereinigten Staaten. Drittens wird ihnen von den genannten AutorInnen eine gemeinsame stilistische Ausrichtung zugeschrieben, die sich an Stilmitteln der New Wave bzw. des Postpunk orientiere, was sich viertens in den Besetzungen der Bands niederschlägt: in der Regel Quartette in klassischer Rockformation (dr, b, 1-2 git, voc), mitunter zum Trio reduziert oder um einen Keyboarder zum Quintett erweitert. Fünftens spielen in diesen Bands zum allergrößten Teil weiße Männer, die zum Teil deutlich jünger als dreißig Jahre sind. ${ }^{3}$ Als letztes Merkmal vertritt keine der Bands eine ausdrücklich politische Haltung, wie es einige der New Wave- oder Postpunk-Vertreter der 1980er Jahre taten. ${ }^{4}$

Das hier im Mittelpunkt des Interesses stehende Hauptmerkmal dieser Gruppe ist jedoch, dass die Rezipienten in ihren Feststellungen über die Musik dieser Bands eine gemeinsame musikalische Abstammungslinie zu erkennen glauben, d.h. die Musik auf ein »Ur-Modell« zurückführen. Die in den Medien immer wieder genannten Namen der »Referenz-Bands « (Scharlau/Venker/Steinbrink 2005: 40) lassen sich zu drei Generationen zusammenfassen, von denen zwei in engerem zeitlichen Abstand liegen. Als direktes Vorgängermodell dieser Bands wird die Formation Franz Ferdinand genannt: vier weiße junge Männer aus Großbritannien, die selber erst 2004 ihr Debütalbum veröffentlichten und spätestens seit dem Erscheinen ihres zweiten Albums 2005 als etabliert gelten. Innen ging in der popjournalistischen Geschichtsschreibung mit der US-amerikanischen Band The Strokes eine zweite Generation voraus, die 2001 debütierte und seitdem als Vorrei-

2 Vgl. bspw. Behr/Leimann/Flore 2005; Drüner 2005; Fuchs 2005; Goodwin 2005; Hentschel 2005; Hildebrand 2005; Kedves 2005; Kubiak 2005; Kubriak 2005; Scharlau 2005; Stoppenhagen 2005; Winkler 2005; Wohlfeld 2005; Ziemer 2005a, 2005b, 2005c.

3 Einzige Ausnahmen sind meines Wissens der farbige Sänger von Bloc Party und die deutschstämmige Bassistin von Art Brut.

4 Ausdrücklich politischer Protest, wie inn beispielsweise The Beat als Vertreter des Postpunk gegen die Regierung Thatchers formulierten (vgl. Walter 2005: 30 ), oder eine ähnliche Politisierung wie die marxistisch-anarchistisch inspirierte politische Agenda der Band Gang of Four (vgl. Winkler 2005) findet sich allerhöchstens bei Bloc Party; der Sänger thematisiert in seinen Texten Probleme wie Armut und soziale Kälte. 
ter und Messlatte einer großen Menge junger Rockbands mit dem bestimmten englischen Artikel im Namen gilt. Jeder der beiden Bands wird eine Vorbildfunktion für die beschriebene Gruppe zugeschrieben. ${ }^{5}$ Die erste Generation dagegen setzt sich aus Bands zusammen, die in den 1970er und 1980er Jahren ihre ersten Singles und Alben veröffentlichten. Zu ihnen werden Gang of Four, Talking Heads, Wire, The Smiths, The Cure, The Jam und andere gezählt.

Diese als Muster '05 zusammengefassten Eigenschaften der Bands sowie die im Popmusikdiskurs erfassten Aussagen, in Reineckes Worten "Sachverhalte der und Feststellungen über Musik«, tragen die Aufgabe der Suche nach Zusammenhängen an die Musikwissenschaft heran. Die leitende Fragestellung dieses Textes wird im Folgenden sein, ob und wie man als popmusikalisch versierter Musikwissenschaftler mit der musikalischen Analyse »Rock, Pop, Jazz musikimmanent durchleuchte[n]« (Rösing 1989) kann, um nachvollziehbar zu machen, mithilfe welcher Gestaltungsmittel populäre Musik geformt ist, die im öffentlichen Diskurs eine derart übereinstimmende Beschreibung erfährt. Somit wäre einerseits zumindest ein Anschluss an aktuelle Themen des Popmusikdiskurses unternommen, andererseits die Reichweite und Möglichkeit von Analyse, der vermeintlichen Königsdisziplin der Zunft, in concreto untersucht.

Der eher als marginal zu bezeichnende Beitrag der Musikwissenschaft zu dem, „was >musikalisch passiert«, veranlasste Reinecke (1992: 12) zu der Vermutung, dass den "geängstigten Musikologen« für die Auseinandersetzung mit »Popularmusik « ihr Rüstzeug nicht ganz ausreiche. Ansgar Jerrentrup (1989: 134) gab zwar zu bedenken, dass eine »rein musikimmanente Betrachtung « Jazz, Rock und Pop nicht zufriedenstellend erklären könne, plädierte nichtsdestotrotz, eine Bresche für die Disziplin schlagend, für die »überwiegend musikimmanente Betrachtung « dieser Musik. Derart äußert sich auch Martin Pfleiderer, der einen systematisierenden Zugriff auf die Gestaltungsmittel populärer Musik vorstellt - mit dem Hinweis, dass einem »Verständnis populärer Musikformen« (Pfleiderer 2003: 18) ohne ein umfassendes Ineinandergreifen verschiedenster Disziplinen nicht gedient sei.

Im Bewusstsein dieser fachinternen Einwände wird nachfolgend trotzdem das Instrumentarium der musikalischen Analyse als Ausgangspunkt der

5 So seien Maxïmo Park eine »exakte Mischung aus Franz Ferdinand und The Strokes" (Scharlau 2005), die Kaiser Chiefs dagegen nur eine "Strokes-Nachäfferband«, denen »Image-Kalkül« unterstellt wird (Hentschel 2005: 95). Wie man sieht: die Erfüllung des Musters '05 mag gelingen oder nicht; diese stellvertretenden Zitate belegen dennoch das offensichtliche Existieren solch formativer Regeln und ästhetischer Konzepte als Bedingung für einen Bereich popmusikalischer Praxis im Jahr 2005. 
Auseinandersetzung mit der Musik Maxïmo Parks stellvertretend für die Gruppe der 2005er-Bands genutzt. Denn ein sich als musikwissenschaftlich verstehender Zugang zur Musik muss sich im Vergleich zu anderen Ansätzen, ob semiotisch, poststrukturalistisch, marxistisch oder soziologisch inspiriert, seiner spezifischen Werkzeuge bewusst werden und diese am musikalischen Material auf ihre Nutzbarkeit überprüfen. Erst auf der jeweils unterschiedlichen materiellen Basis, die hinreichend nur von musikwissenschaftlicher Seite erfasst werden kann, kann Musik im Kontext ihrer Verwendung eine Bedeutung erfahren. Welche das im Einzelfall ist, kann allerdings nur diejenige Analyse beschreiben, die ihren Objektbegriff um die Untersuchung des Kontextes erweitert.

\section{Maximo Park}

Maxïmo Park gründeten sich Ende 2002 im englischen Newcastle und spielen seitdem in Quintettbesetzung mit Sänger Paul Smith, Duncan Lloyd an der Gitarre, Archis Tiku am Bass, Lukas Wooller am Keyboard sowie Schlagzeuger Tom English. Durch ihre erste selbstproduzierte und -vertriebene Single in 500er-Auflage wurde Rough Trade, eines der wichtigsten und größten Labels und Vertriebe des Independentsektors, auf die Band aufmerksam und nahm sie in den Vertrieb auf. Mit dieser prominenten Unterstützung bekam die Band einen Live-Auftritt bei BBC-Six, woraufhin sich die vornehmlich als Elektroniklabel bekannte Plattenfirma Warp Records interessiert zeigte. Es kam zu einem Wettbieten mehrerer Labels um den Vertrag mit Maxïmo Park (vgl. Scharlau/Venker/Steinbrink 2005: 39f.). Die Band unterzeichnete schließlich bei Warp und veröffentlichte drei weitere Singles, bevor ihr Debütalbum A Certain Trigger (Maxïmo Park 2005a) im Mai 2005 erschien. Das Album erreichte Platz 15 in den englischen Charts. Im selben Jahr noch brachte Warp mit Missing Songs (Maxïmo Park 2005b) ein Album mit B-Seiten und Demoversionen der Singles auf den Markt.

Sämtliche oben genannten verbindenden Eigenschaften des Musters '05 sind bei Maxïmo Park erkennbar: die Punkte Debütalbum, Herkunftsland, zugeschriebener Stil und Besetzung entsprechen den Kategorien genauso wie der Umstand, dass die Bandmitglieder durchweg junge (nur von Paul Smith ist das Geburtsjahr, 1979, zu erfahren) männliche Weiße sind. Zudem vertritt die Band laut Sänger Paul Smith keine ausdrücklich politischkritische Haltung:

»Ich halte es für schwierig zu sagen, man sei eine politische Band. Auch kann ich nicht wirklich behaupten, dass Politik in unserer Musik explizit verarbei- 
tet wird. [...] Ich kann mich höchstens dazu durchringen, zu sagen, dass unsere Musik eine vereinende Kraft haben soll. [...] Ich singe über Liebe im Alltag oder Liebe, die verflogen ist« (Paul Smith, zit. n. Scharlau/Venker/ Steinbrink 2005: 40).

Wie klingt aber nun die Musik von Maxïmo Park, welches sind die herausragenden und übergreifenden musikalischen Gestaltungsmittel? Produziert wurde Maxïmo Parks erstes Album von Paul Epworth, der ebenfalls für die Langspieldebüts von Bloc Party und The Futureheads, zwei andere Bands der New-New-Wave-Gruppe, verantwortlich zeichnet. Allen drei Alben gemeinsam ist ein stark komprimierter, sehr präsenter und druckvoller Sound, der besonders in den Gitarren mit vielen Höhen angereichert ist. A Certain Trigger enthält dreizehn Songs, die von allen Mitgliedern bis auf Schlagzeuger English in verschiedenen Konstellationen komponiert wurden. Mit 39 Minuten ist die Gesamtspiellänge des Albums dennoch erstaunlich kurz, die meisten Songs sind zwischen zwei und dreieinhalb Minuten lang und ihr Tempo liegt vorwiegend im Bereich zwischen 150 und 170 bpm. Alle Songs stehen im Viervierteltakt.

In mehrerlei Hinsicht fällt allein »Acrobat«, der vorletzte Track, aus dem Rahmen: Er ist der mit Abstand längste Song auf dem Album (4:42 min.); er ist der langsamste des ganzen Albums (94 bpm); der Viervierteltakt wird normal betont und ohne rhythmische Extravaganzen dargeboten; er durchbricht die Instrumentierungsnorm des Albums (Drumcomputer statt Schlagzeug, stark verzerrte, mit viel Hall gemischte und in den Höhen reduzierte E-Gitarren, lang gehaltene akkordische Keyboardflächen); in den Strophen spricht der Sänger den Text, erst im Refrain setzt die Gesangslinie ein. Die Harmonien bewegen sich in einem einfachen Rahmen, in den Strophen wiederholt sich eine viertaktige I-IV-Sequenz (in der vierten Wiederholung I-IIm), der Refrain benutzt die Akkordfolge VIm-IV-IIIm-IV. ${ }^{6}$ Eine Erklärung für dieses einzige Abweichen von der musikalischen Textur der übrigen Songs bietet Paul Smith in einem Interview (Schumacher 2005): Dieser Song sei der emotionalste des Albums, live wolle man den Song nie spielen und er sollte eigentlich auch nicht veröffentlicht werden, da man eine »up-beat pop record« herausbringen wollte. Erst auf Druck der Plattenfirma sei »Acrobat « integriert worden.

6 Auch wenn die Harmonien in einen funktionsharmonischen Rahmen zu passen scheinen, möchte ich davon absehen, sie nach der bekannten Riemannschen Nomenklatur (T, S, D usw.) zu benennen, da hier keine logischen kompositorischen Schritte erkennbar sind, die eine derartige Kennzeichnung rechtfertigen - und die zudem für einen völlig anderen musikalischen Zusammenhang erdacht wurde. 
Diese Erklärung für den eigentümlichen Bruch mit der intendierten einheitlichen ästhetischen Gestaltung ihres Debütalbums leitet über zu der Frage, womit Maxïmo Park das angestrebte Ideal der »up-beat pop record « bei den übrigen zwölf Songs erreichen. Zunächst fällt das durchweg höhere Tempo der Songs sowie deren Kürze auf. Zum schnellen Tempo kommt eine rhythmische und metrische Lust an der Desorientierung und Beschleunigung hinzu, die vor allem vom Schlagzeug geleistet wird. Kein einziger Song wird in einer einheitlichen Begleitmanier durchgespielt. Es gibt viele, nicht selten überraschend Aufmerksamkeit erregende rhythmische und metrische Effekte in den Songs: beispielsweise beginnt das Schlagzeug in »Going Missing « auf der dritten Zählzeit des Viervierteltakts, so dass der Hörer als Übergang zum nächsten Teil einen 2/4-Takt zu hören glaubt; die Bass-Drum spielt oftmals Viertel durch; unvermutet tauchen ternäre Rhythmen vor binären Metren auf und English benutzt vielseitig an Songteile gebundene Rolls und Abweichungen vom Standardkitpattern (ganze Abschnitte eines Songs werden etwa nur auf den Toms oder ohne Snare-Drum gespielt). Die Instrumentierung der Songs bleibt immer ähnlich: zu hören sind Schlagzeug, Bass, E-Gitarren, ${ }^{7}$ ein Keyboard, das meist Orgel-ähnliche Sounds spielt, sowie die helle, auf charakteristische Weise zugleich kraftvoll und verletzlich klingende Gesangsstimme, die in den Songteilen mit Refraincharakter gedoppelt wird. Selten kommen eine zweite Gesangsstimme oder Backgroundvocals mit Summvokalisen vor.

Die harmonischen Aspekte von Maxïmo Parks Musik zeigen eine klare Abwendung von der Funktionsharmonik hin zu einer Stufenharmonik, ohne dabei einen Modus zu bedienen. Neben der im Rock häufig benutzten I-IVVerbindung finden sich Sekundrückungen und überraschende Sprünge, die auf einem harmoniefremden Akkord landen. ${ }^{8}$ Diese zunächst willkürlich anmutenden Akkordfolgen erscheinen logischer, wenn man, Allan F. Moore (2001: 59f.) folgend, die Kompositionsweise der Songs in die Analyse einbezieht. Bis auf zwei Songs (»I Want You To Stay«, »Acrobat«) wurden alle von dem Gitarristen, dem Bassisten oder dem des Gitarrespielens mächtigen Sänger der Band komponiert, so dass zu vermuten ist, dass instrumentaltechnische und klangästhetische Aspekte im Kompositionsprozess (funktions-)

7 Fast durchweg spielte Gitarrist Lloyd eine zweite E-Gitarrenspur ein, die doppelnde oder kontrapunktierende Funktion hat; bei »Going Missing « erklingt eine Akustikgitarre, welche die E-Gitarrenfigur doppelt.

8 Im Song "Signal And Sign« werden im A-Teil so genannte Powerchords (Grundton, Quinte, Oktave) über den Tönen $h, a$, cis gespielt, im B-Teil geht es ohne Modulation nach G-Dur, dem ein $C^{9}$ folgt, im C-Teil beginnt harmonisch unvermittelt ein E-Dur-Akkord, der von der gezupften Akkordfortschreitung $\mathrm{C}$ - G/h $G-D /$ fis $-G-D /$ fis - E beendet wird. 
harmonische Überlegungen überlagert haben bzw. überhaupt keine Rolle spielten. ${ }^{9}$

Am Beispiel des Songs »Apply Some Pressure« werden diese Punkte deutlich. »Apply Some Pressure«, zweiter Song auf dem Album und erste Hitsingle bei Warp (Platz 20 in den englischen Charts), hat eine Länge von 3:19 min. und ein Tempo von $164 \mathrm{bpm}$. Bereits zu Anfang verwirrt das auf der zweiten Achtel des Taktes beginnende zweitaktige Eröffnungsriff der Gitarre, eine viertönige, auf A-Dur-Pentatonik beruhende Achtelfigur (Phrase a), die von einer Phrase b bzw. b' akkordisch beantwortet wird:

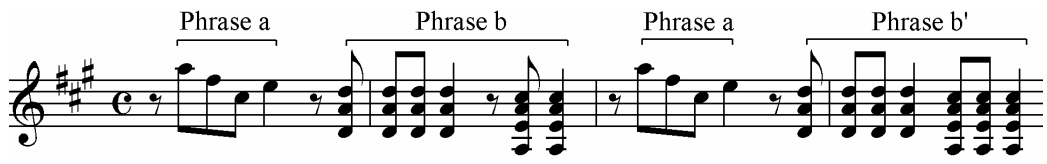

Abbildung 1: »Apply Some Pressure«, Eröffnungsriff

Zusätzlich sorgt die begleitende zweitaktige Schlagzeugfigur mit den Tomrolls und der verschobenen Snarebetonung (im ersten Takt auf der zweiten und dritten Viertel, im zweiten Takt auf der ersten, zweiten und vierten Viertel) für rhythmische Desorientierung. Ein von mir als »Störmotiv« bezeichnetes eintaktiges Zwischenriff unterbricht die Periodizität der Form. Seine Offbeat-Betonung behindert den rhythmischen Fluss, stört aber gleichzeitig auch das bisher etablierte tonale Zentrum auf A-Dur durch Sekundrückungen:

9 Dem A-Teil von "Signal And Sign« mit dem tonalen Zentrum $h$ folgt ein G-DurAkkord, der meiner Deutung nach weniger als mediantische Wendung denn als klanglich überzeugende, im Experiment gefundene Fortschreitung zu werten ist; dergleichen gilt für die Rückung nach E-Dur in Teil C. Dessen Fortführung (s. Fußnote 8) ist vor allem spieltechnischen Aspekten geschuldet, da sie mit nur zwei Fingern gegriffen wird. Andere gitarrenspezifische Gestaltungsmittel bei Maxïmo Park sind etwa Powerchords, typische offen gespielte, d.h. mit Leersaiten gegriffene Akkordfolgen wie $\mathrm{G}-\mathrm{C}^{9}$ oder das »dropped-d-tuning « beim Song "Going Missing «: der Powerchord-Griff der Sekundrückung über den Tönen $e$ und $d$ auf den Saiten $A, d$ und $g$ wird beibehalten und eine Saite tiefer die gleiche Spielfigur ausgeführt. Es erklingt nun die Rückung über den Tönen $a$ und $\mathrm{g}$, diesmal mit hinzugefügter Sexte. Der instrumentale Aufwand ist gering, das klangliche Ergebnis erstaunlich. 


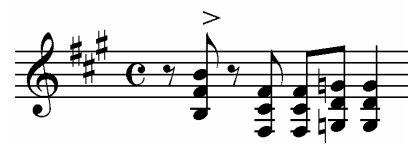

Abbildung 2: »Apply Some Pressure«, Störmotiv

Die rhythmische und harmonische Brechung als Prinzip wird mit dem neuen Riff in Teil B etabliert:

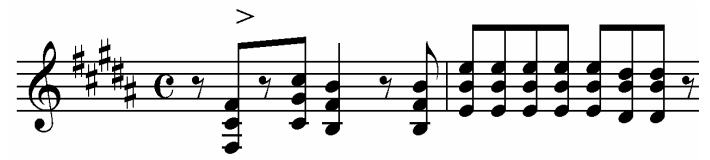

Abbildung 3: »Apply Some Pressure«, Riff Teil B

Auch die folgenden Teile $C$ und $D$ stellen jeweils neues musikalisches Material vor, das neue Harmonien (Teil C: $D^{\mathrm{j} 7}-\mathrm{E}$; Teil D: Hm - D - A - E) und rhythmische Veränderungen beinhaltet. Teil $C$ behält bei Reduktion der Klangdichte die Betonung der zweiten Achtel des Taktes bei, unterstützt durch das alle zwei Takte wiederholte Öffnen der Hi-Hat auf der letzten Achtel des vorangehenden Taktes. Teil D bringt dagegen erstmals eine gleichmäßige rhythmische Textur mit durchgeschlagenen Achteln in Bass, Gitarre und Keyboard, das hier einen Klaviersound spielt. Der Sound des Songs fügt sich in die oben beschriebene klangliche Gestaltung des Albums ein. Schlagzeug, Gitarre und Gesang klingen präsent, in der Tiefenstaffelung sind Bass und Keyboard etwas nach hinten genommen. Die dynamische Dichte ist enorm komprimiert. Selbst in der ruhigen Passage des Teils C bleibt der Klang druckvoll.

Darüber hinaus wird ein weiteres musikalisches Gestaltungsmittel als Charakteristikum der Musik von Maxïmo Park evident, nämlich die mehrteilige formale Anlage (s. Tabelle 1). Hier sind vier Teile harmonisch eindeutig getrennt. Bemerkenswert daran ist ihre unentschiedene Funktion. Eine eindeutig eröffnende Funktion scheint der Teil A zu haben, wohingegen die Teile $B, C$, und $D$ sich gegen eine einfache Zuteilung zu den etablierten Strophen-, Bridge- oder Refrainbezeichnungen sträuben. B ist weder eine Überleitung noch ein Refrain, da die melodische Struktur von A übernommen wird, allerdings vor einem völlig neuen harmonischen Hintergrund. C könnte als Refrain bezeichnet werden, da hier der Songtitel gesungen wird - jedoch sagt uns der verhaltene Charakter dieser Passage sowie der mit Chorus und Hall bearbeitete Gitarrensound, dass es sich hier um eine 
ruhige Überleitung handelt, die zudem mit einer Steigerung endet. Der anschließende Teil D dagegen könnte eher als Refrain verstanden werden, allerdings erst in der Form D', wenn die Backgroundchöre den Text »Apply some pressure, use some pressure « singen und damit den Songtitel nennen. Dagegen spricht wiederum, dass der Song - entgegen allen ungeschriebenen Gesetzen des Popsongs, nach denen man mit dem Refrain aufhört - nicht mit diesem Teil $D$ endet, sondern das Eröffnungsriff wieder aufgenommen wird.

\begin{tabular}{|c|c|c|c|}
\hline Teil & Funktion & Länge & Harmonik \\
\hline $1 / 2 \mathrm{~A}$ & Intro & 4 Takte $(2+2)$ & Riff, tonales Zentrum A \\
\hline \multirow[t]{2}{*}{ A } & Strophe & $\begin{array}{l}8 \text { Takte } \\
(2+2+2+2)\end{array}$ & Riff, tonales Zentrum A \\
\hline & »Störmotiv« & 1 Takt & $H^{5}-F_{i s}^{5}-G^{5}$ \\
\hline$A^{\prime}$ & Strophe & $\begin{array}{l}8 \text { Takte } \\
(2+2+2+2)\end{array}$ & $\mathrm{Hm}-\mathrm{D}-\mathrm{A}$ \\
\hline B & $\begin{array}{l}\text { Strophen-/ } \\
\text { Refraincharakter }\end{array}$ & $\begin{array}{l}8 \text { Takte } \\
(2+2+2+2)\end{array}$ & $\mathrm{Fis}^{5}-\mathrm{Cis}^{5}-\mathrm{H}^{5}-\mathrm{E}^{5}-\mathrm{H} / \mathrm{dis}$ \\
\hline \multirow[t]{2}{*}{ A } & Strophe & 8 Takte & Riff, tonales Zentrum A \\
\hline & »Störmotiv« & 1 Takt & $H^{5}-\mathrm{Fis}^{5}-\mathrm{G}^{5}$ \\
\hline$A^{\prime}$ & Strophe & 8 Takte & $\mathrm{Hm}-\mathrm{D}-\mathrm{A}$ \\
\hline B & $\begin{array}{l}\text { Strophen-/ } \\
\text { Refraincharakter }\end{array}$ & 8 Takte & $\mathrm{Fis}^{5}-\mathrm{Cis}^{5}-\mathrm{H}^{5}-\mathrm{E}^{5}-\mathrm{H} / \mathrm{dis}$ \\
\hline $\mathrm{C}$ & $\begin{array}{l}\text { Bridge-/ } \\
\text { Refraincharakter }\end{array}$ & $\begin{array}{l}9,5 \text { Takte } \\
(4+3,5+2)\end{array}$ & $D^{j 7}-E$ \\
\hline D & $\begin{array}{l}\text { Bridge-/ } \\
\text { Refraincharakter }\end{array}$ & 16 Takte $(8+8)$ & $H m-D-A-E$ \\
\hline C & $\begin{array}{l}\text { Bridge-/ } \\
\text { Refraincharakter }\end{array}$ & 9,5 Takte & $D^{j 7}-E$ \\
\hline D & $\begin{array}{l}\text { Bridge-/ } \\
\text { Refraincharakter }\end{array}$ & 16 Takte & $H m-D-A-E$ \\
\hline$D^{\prime}$ & Refraincharakter & 16 Takte & $H m-D-A-E$ \\
\hline $1 / 2 D^{\prime}$ & Refraincharakter & 8 Takte & $H m-D-A-E$ \\
\hline \multirow[t]{2}{*}{$A^{\prime}$} & Strophe & 8 Takte & $\mathrm{Hm}-\mathrm{D}-\mathrm{A}$ \\
\hline & »Störmotiv« & 1 Takt & $H^{5}-F i s^{5}-G^{5}$ \\
\hline
\end{tabular}

Tabelle 1: »Apply Some Pressure«, Form 
Eine Erklärung für diese verwirrende formale Anlage bietet der Sänger in einem Interview (Schumacher 2005). Man habe bei der Komposition des Songs bewusst eine unkonventionelle Struktur angestrebt, deren verschiedene Teile alle als Refrain gelten sollten. Ziel sei gewesen, diesen Song als Hitsingle im Radio zu platzieren, deshalb habe man auch auf die zweite Strophe (hier: das zweite Erscheinen der Teile A - A' - B) beim so genannten Radio-Edit verzichtet. ${ }^{10}$ Derart lässt sich auch der offensichtliche HookCharakter der Gesangslinie begründen, die sich eng an den Gitarrenriffs orientiert. In den Teilen A, A' und B wird die aus Sprüngen bestehende Phrase a gedoppelt, die Phrase $b$ leicht variiert, ohne den repetitiven Gestus zu verlassen. Das melodische Material beruht auf der Durskala, wobei Grundton und Terz während des Melodieverlaufs häufiger benutzt, Terz und Quinte des jeweiligen Akkordes bevorzugt als letzter Melodieton angesteuert werden. Die angestrebte Aufgabe, vier Teile mit Refraincharakter zu komponieren, macht schließlich auch die uneinheitliche harmonische Textur verständlich. Tatsächlich scheinen die vier Teile dem Wortsinne des lateinischen componere nach »zusammengefügt« worden zu sein.

\section{Ähnlichkeiten und Muster}

Nachdem nun verschiedene musikalische Gestaltungsmittel vorgestellt wurden, die bei Maxïmo Park Verwendung finden, ist die Frage nach den Ähnlichkeiten als eine Vorstufe der Suche nach Mustern wieder aufzunehmen. Und zwar gilt es zu beantworten, warum bei der Beschreibung dieser Musik ein relativ enger Kreis von bekannten Namen als Maßstab und Vergleichsgröße genannt werden. Kurz gesagt: klingen Maxïmo Park wirklich genau wie The Jam, der für Maxïmo Park am häufigsten genannten Referenzband, die hier exemplarisch für die anderen Bands der ersten Generation zum Vergleich herangezogen wird? Können wir gemeinsame musikalische Gestaltungsmittel nachweisen, so dass die öffentliche Wahrnehmung von Maxïmo Park in den Medien aufgrund der Ähnlichkeit der musikalischen Gestaltungsmittel nachvollziehbar ist? Da eine ähnlich aufwendige Untersuchung der Musik von The Jam den Rahmen dieses Textes sprengen würde, greife

10 Im vergleichenden Höreindruck wirkt der 2:44 Minuten lange Radio-Edit verwirrend vielfältiger durch die schnelle Aufeinanderfolge neuer Teile. Nur C und D werden wiederholt, so dass sie sich tiefer ins Gedächtnis einprägen können. Die überraschende Wiederaufnahme des achttaktigen A'-Teils samt Störmotiv kommt einem musikalischen Ausrufezeichen am Ende des Stückes gleich. 
ich im Folgenden auf zwei Analysen von Dirk Budde (1997) und Allan F. Moore (2001) zurück.

Budde (1997: 64f.) beschreibt die Musik von The Jam als motivorientierte und energiegeladene Punkversion mit vielen Soul-Einflüssen, die sich aber in Sound und Verlauf vorwiegend an die frühe Musik von The Who anlehne. Obwohl die Stimmführung sehr melodisch sei, empfinde man die Musik als eckig und abgehackt, was Budde auf die nicht näher bezeichnete Spielweise des Schlagzeugs zurückführt. Ihre Songs seien kraftvoll und schnell, mit geringem Aufwand »stark arrangiert und gut durchdacht « (ebd.: 65). Auch Moore (2001: 142) benutzt das Adjektiv energiegeladen (»highly energized «), um die in seinen Augen im kommerziellen Soul und vor allem Beat der 1960er Jahre verwurzelte Musik von The Jam zu beschreiben. Er weist jedoch darauf hin, dass sich die Band zu Beginn der 1980er Jahre weiterentwickelt habe. Waren vorher »strange rhythmic groupings and comparative freedom from regular verse/chorus structures « $z u$ finden, so benutze die Musik anschließend gängige Formen (»regular groupings«) und herkömmliche Harmonieschemata des »mid-1960s' soul « (ebd.: 143), über die Sänger Paul Weller kämpferische Texte über das Leben der englischen Arbeiterklasse singe. Zur selben Zeit vergrößerte sich die Besetzung des Trios (git, b, dr) zunächst um einen Keyboarder, 1982 schließlich zu einem »full soul line-up« (ebd.). Als Beispielsongs führen beide Autoren »In The City« (1977), »David Watts« (1978) und »Eton Rifles« (1979) an.

Auf dieser Zusammenfassung basierend, scheint sich eine vordergründige Ähnlichkeit der Musik von The Jam mit der Maxïmo Parks feststellen zu lassen: Zunächst ist die Besetzung relativ ähnlich, in beiden Fällen können »motivorientierte« Stellen nachgewiesen werden, in denen die Gitarre bzw. - bei »David Watts« - das Klavier die Gesangslinie doppelt. Auch würde »energiegeladen « die Musik Maxïmo Parks einigermaßen treffend beschreiben. Dennoch fällt es schwer, sich beim Hören dieser Songs The Jam als Maßstab, Vergleichsgröße oder gar Vorbild Maxïmo Parks vorzustellen, denn in den musikalischen Gestaltungsmitteln der beiden Bands lassen sich zwar einzelne Gemeinsamkeiten (schnelles Tempo, Besetzung) erkennen, diese liegen jedoch jenseits dessen, was als musikwissenschaftlicher Indizienbeweis vorgeführt werden könnte; sie treffen auch auf hunderte anderer Bands zu.

In ad-hoc durchgeführten Tests mit Hörern, denen diese drei Songs neben »Apply Some Pressure« ohne Nennung von Titel, Interpret und Jahr der Aufnahme mit der Bitte, auf Ähnlichkeiten zu achten, vorgespielt wurden, kam Folgendes heraus: »Eton Rifles«, die erfolgreichste Single von The Jam, wies für die Hörer am wenigsten, nämlich fast keine Ähnlichkeit 
mit »Apply Some Pressure « auf, gefolgt von »In The City«. Als ähnlicher, keinesfalls identisch wurde »David Watts« empfunden, allerdings gleich ähnlich wie das abschließend vorgespielte »Let's Spend The Night Together « der Rolling Stones - ein Song, der bei gleicher Tonart, Schlagzeug- und Klavierbegleitung sowie dem in Achtelnoten zwischen Grundton und Oktave pendelnden Bass eine fast identische Gesangslinie wie "David Watts« aufweist.

Für unsere Suche nach Ähnlichkeiten ist dieser Befund niederschmetternd: Sollten Maxïmo Park tatsächlich wie The Jam klingen - was wir anhand der wenigen gemeinsamen Parameter nicht schlüssig und ausschlieBend belegen können -, dann klingen sie auch ähnlich wie die Rolling Stones, ein Name, der niemals in den Medien zum Vergleich herangezogen wurde. Dies lässt den Vergleich mit The Jam zunächst als einigermaßen willkürliche Entscheidung wirken.

\section{Von der musikalischen Analyse zur Analyse von Musik}

Das Problem musikalischer Analyse, so wie sie hier bisher eng am Material betrieben wurde, ist einerseits, dass wir keine Aussage über Ähnlichkeiten zwischen zwei Stücken treffen können, die über den Status des musikalischen Zitats hinausgehen. Derartige Allusionen können nicht angemessen in hierarchischen Graden von Gleichheit oder Ungleichheit ausgedrückt werden. ${ }^{11}$ Die Frage, ob »David Watts« oder »Let's Spend The Night Together « einander ähneln, kann in einer herkömmlichen Analyse beantwortet werden. Die Frage jedoch, warum ausgerechnet The Jam mit Maxïmo Park ver-

11 Die Ähnlichkeiten von musikalischem Material zu untersuchen, hat sich das amerikanische Music Genome Project zur Aufgabe gemacht. Es will nichts Geringeres als eine "essence of music at the most fundamental level « (Westergren 2006) erfassen: »It's not about what a band looks like, or what genre they supposedly belong to, or about who buys their records - it's about what each individual song sounds like « (ebd.). Dazu analysieren Musiker und Musikwissenschaftler populäre Musik mithilfe eines Katalogs, der 400 Kriterien umfasst. Auf der von innen betriebenen Website www. pandora.com bieten sie eine Radiostation an, der Hörer mehrere Lieblingsbands und -lieder nennen können. Diese werden daraufhin auf Ähnlichkeiten mithilfe des Kriterienkatalogs und einer nicht näher spezifizierten Rechenoperation untersucht. Anschließend werden neue Songvorschläge vorgespielt, die wiederum vom User bewertet werden können. Bei Angabe des »favorite artist « Maxïmo Park wurden mir völlig unbekannte Bands vorgeschlagen, als Begründung gab man an: "hard rock roots, punk influences, mild rhythmic syncopation, subtle use of vocal harmony, and major key tonality«. The Jam waren nicht darunter. 
glichen werden, die Rolling Stones jedoch nicht - und was das alles mit New Wave oder Postpunk zu tun hat, um den eingangs gestellten Bezug wieder aufzunehmen -, kann mit dem Verweis auf die zweite, viel grundlegendere Schwäche musikalischer Analyse beantwortet werden: Musikalische Analyse, wie sie in der traditionellen Musikwissenschaft gelehrt und betrieben wurde und wird, achtet bis heute nicht hinreichend auf den sozialen, politischen und ökonomischen Kontext, in dem die untersuchte Musik produziert, vertrieben und rezipiert wurde. ${ }^{12}$

Peter Wicke (2004) trifft eine hilfreiche Unterscheidung zwischen "Soundtrack« und »Musik«. Unter Soundtrack versteht er eine »spezielle Daseinsweise von Klang «, die als Dauerbeschallung durch den Alltag ziehe. Erst durch komplexe soziale und kulturelle Prozesse der Bedeutungsverleihung werde dieser Klang zu Musik:

»Orte, Räume und Szenen spielen dafür [für die »Musikwerdung « von Soundtracks, A. D.] ebenso eine Rolle wie Märkte und Medien, Bilder, Worte und Anschauungen, Vorstellungswelten und Ideologien. Als Musik erscheinen solche Tonspuren im Alltag nur dann, wenn sie vom hörenden Subjekt aufgenommen und in die Geographie eines individuell gelebten Lebens übersetzt sind « (Wicke 2004: 117).

Musikalische Analyse, die sich ausschließlich auf die Parameter der musikalischen Gestaltung beschränkt, beschäftigt sich demzufolge vorwiegend mit Soundtracks, denen sie eine besondere, intradisziplinär gültige Musikwerdung zukommen lässt. Es werden Akkorde benannt, Formschemata erstellt usw., die für die Fachöffentlichkeit bedeutend sein mögen - nur ist sie auf diese Weise nicht in der Lage, die öffentliche Bedeutung der von ihr analysierten Musik im popularmusikalischen Diskurs zu erkennen. ${ }^{13}$ Demgegenüber gehört zur Analyse von Musik im Sinne Wickes unabdingbar, sich mit der Produktion von Bedeutungen zu befassen, die bei der Musikerfahrung generiert werden. Wicke stellt klar, dass Musik kein Container sei, der Bedeutungen enthält, die nach Belieben wieder entnommen werden können. Sie entstehe in einem Diskurs, der um die Begriffe musikalischer Erfahrung als »Raster oder Filter der Wirklichkeit« (ebd.: 118) geführt wird. Begriffe, wie

12 Obwohl für Gerold W. Gruber (1994: 577f.) im Vordergrund musikalischer Analyse nach wie vor der Zugang zum Kunstwerk steht, »der im Beschreiben des musikalischen Materials, der Strukturelemente, sowie harmonischer und formaler Kriterien liegt «, nimmt auch er zur Kenntnis, dass »neuerdings « (ebd.) Phänomenen wie Wirkungsgeschichte oder Rezeptionsästhetik besondere Aufmerksamkeit zukomme.

13 Hierzu schreibt Dietrich Helms (2002: 100): »Die Musikwissenschaft sollte sich bewußt sein, daß sie >Wahrheit nur für die Teilnehmer des eigenen Diskurses formulieren kann.« 
etwa New Wave, seien umkämpft, da sie sich einerseits als Stilprinzipien gerieren, andererseits jedoch als Marketingkonzepte und zur Identifikation von Zielgruppen benutzt würden, da sich in ihnen soziale, kulturelle, wirtschaftliche, mediale und ideologische Faktoren vernetzen.

»Mit der Vorspiegelung, es ginge um Stil, obwohl Aspekte der Klanggestalt, mithin musikalische Gestaltungskonzepte allenfalls ein Moment in den immer wieder neu hergestellten symbiotischen Beziehungen auf dem kulturellen Terrain darstellen, wird suggeriert, dass die Dinge so, wie sie heißen, auch seien« (Wicke 2004: 117).

Die Ausgangsfrage muss also dahingehend verfeinert werden, dass es nicht um das Erkennen von Mustern im musikalischen Material gehen kann, sondern darum, die Frage der Bedeutungsproduktion als wesentlichen Bestandteil einer Analyse von Musik zu integrieren. Wie kommt also die öffentliche Bedeutung von Maxïmo Park als New Wave-Band in der Nachfolge von The Jam zustande?

Wicke weist darauf hin, dass als zentraler Bestandteil des popmusikalischen Diskurses das strategische Positionieren und Normieren von Begriffen, also die Macht zur Definition als entscheidender Faktor musikalischer Praxis angesehen werden müsse (vgl. ebd.: 121). Deshalb investieren Plattenfirmen in Promotion-Abteilungen, die nicht nur ein visuelles Konzept einer Gruppe erarbeiten und beschließen, wann und wie sie präsentiert werden, sondern auch auf so genannten Waschzetteln Kategorien setzen und besetzen, die, einmal im Diskurs präsent, kaum wieder zu eliminieren sind.

Im Falle Maxïmo Parks sind etliche Bemühungen zu erkennen, das Auftreten der Band auf dem Markt zu steuern. Zunächst ist auffällig, dass ungeheuer großer Wert auf die visuelle Gestaltung von Band und CD gelegt wurde; im Falle einer Firma würde man treffenderweise von der Etablierung eines Corporate Design sprechen. Sämtliche Singleveröffentlichungen, die beiden Alben und der Internetauftritt haben eine einheitliche Typografie und Bildsprache. Letztere zeigt junge Männer und Frauen in Businessanzügen und -kostümen, die beim ekstatischen Tanz, den Kopf abgewandt, schwarzweiß fotografiert wurden. ${ }^{14}$ Im Inneren des Albumcovers sind in der Art der Do-it-Yourself-Ästhetik des Punk die Songtexte, von einer kaputten Schreibmaschine getippt, auf zerknülltem Packpapier geschrieben und dann

14 Paul Smith erzählt in einem Interview (Tepel 2005: 46), dass das Grafikdesignbüro Yes die Optik in Anlehnung an Bilder des Fotografen Robert Longo vorschlug. Darüber hinaus knüpft die Gestaltung an eine andere populäre Zeichensprache an, nämlich die scherenschnittartige Ästhetik der iPod-Werbekampagne von Apple. Auch dort wird der Dynamiktransfer von der Abbildung musikinduzierter Bewegung auf ein Objekt versucht. 
als Faksimile abgedruckt; Teile davon wurden tatsächlich mit Bleistift und Kugelschreiber nachträglich korrigiert, um die Authentizität des Handgemachten visuell zu bekräftigen. Entgegen aller fehlenden politischen Inhalte der Liedtexte signalisieren Schrift- und Farbwahl des Covers (rot, weiß, schwarz) sowie der Umlaut auf dem i des Bandnamens, der an den »Maximo Lider « Fidel Castro erinnert, eine linkspolitische Konnotation. ${ }^{15}$

Auch in den Songtexten und dem Auftreten der Band ist eine große Lust am Spiel mit Bedeutungen zu finden: Zeilen aus anderen Kontexten werden in die Songs wie Slogans eingebaut; etwa wenn auf das Oscar Wilde-Zitat »I am young and I am lost « Smiths Eingeständnis »every sentence has its cost« folgt. Dergleichen ist das Auftreten der Band in Anzügen mit Krawatten ein visueller Verweis auf die ersten Jahre des New Wave; hier ist wahrscheinlich der stärkste Bezug zu The Jam zu sehen, die ähnlich gekleidet auftraten.

Neben dieser mannigfaltigen Bedeutungsproduktion sorgte eine perfekt geplante Veröffentlichungspolitik des Labels für entsprechende mediale Aufmerksamkeit. Bereits im November 2004 wurden Vertreter der internationalen Presse in einen Londoner Club zum Konzert einer relativ unbekannten Band geladen, die bisher nur eine Single veröffentlicht hatte. ${ }^{16}$ Ihnen wurde signalisiert, dass dort die nächste große Band nach Franz Ferdinand auf sie warte. Vertreter der bundesdeutschen Musikpresse vor Ort waren Felix Scharlau von Intro (Scharlau 2005; Scharlau/Venker/Steinbrink 2005) und Uwe Viehmann (2005) von Spex. In beiden Magazinen waren fortan verschiedene Aktivitäten zu beobachten, die als mediale Unterstützung der Band gewertet werden dürfen; der berichtende Musikjournalismus verwandelte sich auf die Stufe der »Schreibe « in Fanzines zurück (Intro ist aus einem Fanzine entstanden). Bereits in den Jahrescharts der Spex-Mitarbeiter für das Jahr 2004 taucht auf Platz 11 ein Song von Maxïmo Park auf (Spex 2005: 71), der zu diesem Zeitpunkt noch gar nicht offiziell in Deutschland veröffentlicht und in England nur als Vinylsingle erhältlich war. Intro verpflichtete Maxïmo Park für Konzerte im Februar 2005 in Deutschland, über die dann in der Märzausgabe enthusiastisch berichtet wurde (vgl. Heitbaum/Steinbrink 2005). Als schließlich im Mai 2005 das Debütalbum er-

15 Auf der Homepage ihrer Plattenfirma erfährt man zu diesem Spiel mit Bedeutungen augenzwinkernd: »The original Maximo Gomez Park is in Havana, Cuba, although there is now another similarly named picnicking [sic] spot in St Petersburg, Florida. The relevance of this fact remains, however, unclear « (Warp Records 2005).

16 Uwe Viehmann (2005: 14) weist darauf hin, dass zu diesem Zeitpunkt immerhin in Großbritannien bereits »jeder gut informierte und -frisierte [sic] Popspatz « über Maxïmo Park Bescheid gewusst habe. 
schien, hatten sowohl Spex als auch Intro Maxïmo Park auf dem Magazincover samt eines mehrseitigen Berichts im Innenteil sowie euphorischer CDBesprechungen (vgl. Fuchs 2005; Hablizel 2005). Intro stellte eine ganze Seite mit Berichten zu Maxïmo Park und dem Album online (vgl. Schwarz 2005). In der Spex war zusätzlich ein Song auf der dem Heft beigelegten CD vertreten. ${ }^{17}$

Doch bereits in diesen ersten Berichten über das Pressekonzert im November 2004 fallen die entscheidenden Begriffe: Scharlau (2005) prophezeit, dass die dort aufgetretene "Sensation« - eine »exakte Mischung aus Franz Ferdinand und den Strokes « - die Band des Jahres werde. Bei Viehmann werden ebenfalls Franz Ferdinand zum Vergleich herangezogen, er aber sieht in Maxïmo Park bereits »eine Mischung aus viel The Jam, dezentem Punkrock-Druck, Powerpop und atmosphärischer Eindringlichkeit à la Interpol« (Viehmann 2005: 14). Dies ist die erste mediale Nennung des Bezugs Maxïmo Park/The Jam in einem bundesdeutschen Leitmedium des Musikjournalismus.

Dass es nun von dieser Feststellung bis zum Terminus »New Wave « nicht mehr weit war, mag damit zusammenhängen, dass der Begriff bereits in den späten 1970er Jahren keine einheitliche Kennzeichnung für eine eng abgrenzbare Gruppe von Interpreten und Bands erlaubte. David Buckley (2001: 818) spricht von einem »imprecise signifier «, Peter Gammond (1991: 421) umreißt inn als einen »vague term« und Thomas Lau (1992: 35) schließlich beklagt, dass unter diesem »unglücklichen Begriff « von den Gothics über die New Romantics und Existentialisten bis hin zu Punkern und Poppern eine extrem heterogene Gruppe zusammengefasst werden konnte - und weiterhin wird. Die Angaben über typische New Wave-Bands reichen dementsprechend auch von den Dire Straits oder Joe Jackson (Gammond) über die Boomtown Rats, Blondie und Talking Heads (Buckley) bis hin zu Devo und D.A.F. (Lau). Insofern sagt die Etikettierung als New Wave-Band oder gar $\gg$ New Wave of New Wave «-Band (Gross 2005) ${ }^{18}$ weniger etwas über inhärente Eigenschaften von Maxïmo Parks Musik als über die Versuche der Musikindustrie, Be-

17 Der deutsche Rolling Stone war etwas zurückhaltender in der Berichterstattung, nahm die Band jedoch trotzdem auf seine CD-Beilage der Maiausgabe. Zusätzlich erschienen in vielen Feuilletons überregionaler Tages- und Wochenzeitungen bis hin zum Magazin der Deutschen Bahn (vgl. von Klot 2005) Berichte und Rezensionen, die allerdings nicht kausal auf das Novemberkonzert bezogen werden können.

18 Buckley (2001: 818) weist darauf hin, dass der fast identische Begriff "new wave of the new wave bereits 1993 von der englischen Musikpresse benutzt wurde, um Bands wie Elastica, $S^{*} M^{*} A^{*} S^{*} H$ und These Animal Men zu kategorisieren, da ihre Musik (wieder einmal) eines tue: »recreating the energy of late1970s punk« (ebd.). 
griffe als Instrumente der Grenzziehung auf dem kulturellen Terrain des Musikmarktes zu benutzen. Nicht nur schafft man derart eine Distanz zwischen sich und dem, was im Mainstream der Charts läuft (und in dem man dennoch vertreten sein möchte), sondern stellt sich zugleich in die (im Übrigen sehr erfolgreiche) Deutungstradition der Vorgängergeneration - auch bei Franz Ferdinand wurde der Begriff »New Wave « als Kategorie benutzt. Dass im Jahr 2005 mit der Strategie, Musik als New Wave zu titulieren, viel Geld zu verdienen war, sprach sich schließlich sogar bis zur ersten Generation herum: Gang of Four (2005) brachten nach über zehn Jahren wieder ein Album auf den Markt, auf dem Neu-Interpretationen alter Songs zu hören sind.

Zusammenfassend lässt sich festhalten, dass die eingangs gestellte Aufgabe, Muster der populären Musik zu untersuchen, nur ein Scheitern zur Folge haben kann, wenn nicht von der musikalischen Analyse ausgehend der Analysebereich um die auf vielen Feldern der musikalischen Praxis stattfindende Bedeutungsproduktion erweitert wird. Einige dieser Felder wurden hier angedeutet und keinesfalls erschöpfend diskutiert. Für zukünftige Forschung besonders wichtig erscheint mir die Untersuchung der Bedeutungsproduktion durch die Musikpresse, da sie für viele Hörer der hier vorgestellten Musik neben dem Internet erste Kontextualisierungsinstanz neuer Musik ist. Freilich darf darüber die musikalische Analyse als Betrachtung der musikalischen Gestaltungsmittel nicht ins Hintertreffen geraten. Denn ihre Aufgabe ist und bleibt es zu zeigen, auf welcher materialen Basis die Produktion von Bedeutungen möglich wird.

\section{Literatur}

Behr, Heiko / Leimann, Eric / Flore, Peter (2005). "New school meets old school, oder die klingen doch wie...«In: Intro, Nr. 129, S. $34 \mathrm{f}$.

Buckley, David (2001). "New wave."In: The New Grove Dictionary of Music and Musicians. Bd. 17. Hg. v. Stanley Sadie. London: Macmillan, S. 818.

Budde, Dirk (1997). Take three chords... Punkrock und die Entwicklung zum American Hardcore (= Schriften zur Popularmusik 2). Karben: Coda.

Dath, Dietmar (2005). "Die wo so singen tun, wie sie der Schnabel gewachst hat.« In: FAZ vom 10. Februar, Nr. 34, S. 37.

Drüner, Henrik (2005). »Kaiser Chiefs. Employment« [CD-Kritik]. In: Intro, Nr. 130, S. 103.

Fuchs, Marco (2005). »Maximo Park. A Certain Trigger « [CD-Kritik]. In: Intro, Nr. 127, S. 112.

Gammond, Peter (1991). The Oxford Companion to Popular Music. Oxford, New York: Oxford University Press. 
Goodwin, Cat (2005). »Maximo Park. A Certain Trigger.«In: NME online, www.nme. com/reviews/maximo-park/7657 (Zugriff: 29.6.2006).

Gross, Thomas (2005). „Die Welle der Welle der Welle. Maximo Park sind die Band des Sommers. «In: Die Zeit vom 2. Juni, Nr. 23, S. 64.

Gruber, Gerold W. (1994). "Analyse."In: Die Musik in Geschichte und Gegenwart. Sachteil, Bd. 1. Hg. v. Ludwig Finscher. Kassel, Stuttgart: Bärenreiter/Metzler (2. Aufl.), Sp. 577-591.

Hablizel, Markus (2005). »Maximo Park. A Certain Trigger« [CD-Kritik]. In: Spex 287, Nr. 5, S. 90.

Heitbaum, Hendrik / Steinbrink, Christian (2005). "Intro Intim. Newcastle meets Prenzlauer Hill.« In: Intro, Nr. 125, S. 144.

Helms, Dietrich (2002). „Musikwissenschaftliche Analyse populärer Musik?« In: Musikwissenschaft und populäre Musik. Versuch einer Bestandsaufnahme. Hg. v. Helmut Rösing, Albrecht Schneider und Martin Pfleiderer. Frankfurt/M. u. a.: Peter Lang, S. 91-103.

Hentschel, Joachim (2005). »Kaiser Chiefs. Employment« [CD-Kritik]. In: Rolling Stone [deutsche Ausgabe], Nr. 8, S. $95 \mathrm{f}$.

Hildebrand, Katrin (2005). "Schrammeln als Kunst. Neue Gitarrenbands auf der Flucht vor dem Immergleichen: Bloc Party, Maximo Park und Weezer.« In: Frankfurter Rundschau vom 20. Mai, Nr. 115, S. 17.

Kedves, Jan (2005). »The Futureheads. Die neuen Herren Gesangsverein. «In: Intro, Nr. 128, S. 52 f.

Klot, Kristina von (2005). „Die Seele aus dem Leib gerockt. Maximo Park werden als die zukünftig beste Band der Welt gehandelt. Mit ihrem Debütalbum >A Certain Trigger s sind sie jetzt live zu erleben. «In: Mobil, H. 11, S. 92.

Kubiak [sic], Jeffrey (2005). »The Futureheads. The Futureheads « [CD-Kritik]. In: Intro, Nr. 128, S. 109 f.

Kubriak [sic], Jeffrey (2005). »Kaiser Chiefs. Restart. «In: Intro, Nr. 129, S. $32 f$.

Lau, Thomas (1992). Die heiligen Narren. Punk 1976-1986. Berlin, New York: de Gruyter.

Moore, Allan F. (2001). Rock. The Primary Text: Developing a Musicology of Rock. Aldershot: Ashgate (2. Aufl.).

Pfleiderer, Martin (2003). "Gestaltungsmittel populärer Musik. Versuch einer Systematik. «In: Samples 2, S. 18-29, www.aspm-samples.de (Zugriff: 29.6.2006).

Reinecke, Hans-Peter (1971). »Naturwissenschaftliche Grundlagen der Musikwissenschaft. "In: Einführung in die systematische Musikwissenschaft. Hg. v. Carl Dahlhaus. Laaber: Laaber, S. 9-51.

Reinecke, Hans-Peter (1992). »Popularmusik und geängstigte Musikologen.« In: Rock / Pop / Jazz im musikwissenschaftlichen Diskurs. Ausgewählte Beiträge zur Popularmusikforschung (= Beiträge zur Popularmusikforschung, Sonderband). Hg. v. Bernd Hoffmann, Winfried Pape und Helmut Rösing. Hamburg: Coda, S. 5-14.

Rolling Stone (2005). »New Noises." In: Rolling Stone [dt. Ausgabe], Nr. 6, S. $34 \mathrm{f}$.

Rösing, Helmut (Hg.) (1989). Rock, Pop, Jazz - musikimmanent durchleuchtet (= Beiträge zur Popularmusikforschung 7/8). Hamburg: Coda.

Scharlau, Felix (2005). »Maximo Park, The Chalets. The Future is Retro. In: Intro. de vom 24. Februar, www.intro.de/magazin/musik/23015382 (Zugriff: 29.6.2006).

Scharlau, Felix / Venker, Thomas / Steinbrink, Christian (2005). "Maximo Park. Langzeitstudie. «In: Intro, Nr. 127, S. 38-40. 
Schumacher, Carsten (2005). »Maximo Park. Track by Track. « [Interview mit Paul Smith und Lukas Wooller, April 2005] In: Intro.de, www.intro.de/musik/ audiovideo/audio/1115202454 (Zugriff: 11.10.2005).

Schwarz, Markus (2005). »A Certain Trigger. Microsite zu Maximo Park. «In: Intro.de vom 22. April, www.intro.de/news/23007614 (Zugriff: 29.6.2006).

Spex (2005). »Mitarbeitercharts 2004.«In: Spex 284, Nr. 1-2, S. 71.

Stoppenhagen, Till (2005). »The Rakes. Capture [CD-Kritik]. In: Intro, Nr. 130, S. $111 \mathrm{f}$.

Tepel, Oliver (2005). »Maximo Park. Rettung im Anzug. « In: Spex 287, Nr. 5, S. 4246.

Viehmann, Uwe (2005). "Maximo Park. The Herzvergabe is always changing." In: Spex 284, Nr. 1-2, S. $14 f$.

Walter, Klaus (2005). »History-Remixer. Der britische Musiktheoretiker Simon Reynolds erzählt seine spannende Version des Post-Punk«. In: Rolling Stone [dt. Ausgabe], Nr. 8, S. 30f.

Warp Records (2005). »Maximo Park. Artist Info. In: Warp Records online, www.warprecords.com/artists/biog.php?artist=mp (Zugriff: 29.6.2006).

Westergren, Tim (2006). »The music genome project. «In: Pandora, www.pandora. com/mgp.shtml (Zugriff: 29.6.2006).

Wicke, Peter (2004). "Soundtracks. Popmusik und Pop-Diskurs." In: Was ist Pop? Zehn Versuche. Hg. v. Walter Grasskamp, Michaela Krützen und Stephan Schmitt. Frankfurt/M.: Fischer, S. 115-139.

Winkler, Thomas (2005). »Britpop-Helden. Rückkehr der Viererbande.«In: SpiegelOnline, www.spiegel.de/kultur/musik/0,1518,380501,00.html (Zugriff: 20.10. 2005).

Wohlfeld, Carsten (2005). »Mit dem Finger am Abzug. Die Rache der Nerds: Auch jenseits des Rock'n'Roll-Hedonismus lassen sich furiose Songs schreiben. Das beweisen Maximo Park auf ihrem Debütalbum. «In: Rolling Stone [dt. Ausgabe], Nr. 5, S. 12.

Ziemer, Jürgen (2005a). »Maximo Park. A Certain Trigger« [CD-Kritik]. In: Rolling Stone [dt. Ausgabe], Nr. 5, S. 109.

Ziemer, Jürgen (2005b). »Rock'n'Roll im Akkord. Die Kaiser Chiefs haben ihre letzte Chance zum Ruhm genutzt. «In: Rolling Stone [dt. Ausgabe], Nr. 8, S. 12.

Ziemer, Jürgen (2005c). »Hard-Fi. Stars of CCTV « [CD-Kritik]. In: Rolling Stone [dt. Ausgabe], Nr. 8, S. $100 f$.

\section{Diskographie}

Gang of Four (2005). Return The Gift. V2 Records, VVR1034432.

Maxïmo Park (2005a). A Certain Trigger. Warp, WARPCD130.

Maxïmo Park (2005b). Missing Songs. Warp, WARPCD142.

Rolling Stones (1967). »Let's Spend The Night Together.« Auf: Between The Buttons. Decca, 820 138-2.

The Jam (1977). »In The City.« Auf: In The City. Polydor, 537417-2.

The Jam (1978). »David Watts. « Auf: All Mod Cons. Polydor, 537419-2.

The Jam (1979). »Eton Rifles. « Auf: Setting Sons. Polydor, 537420-2. 


\begin{abstract}
In this paper the value and range of musical analysis is under discussion. By turning to current popular music of the English band Maxïmo Park, a leading representative of the so-called "new wave of new wave « in 2005, it is suggested that the analytical tool must be seen as a first step in understanding the band's meaning in popular discourse. It is argued that the production of the meaning of a certain piece of music in the discourse is not arbitrary but tied to the song's musical basis in a way analysis is not yet able to specify. Furthermore, the meaning of a piece of music is shaped by the band's representation in the musical press, which must hence be taken into account for the scope of the analysis.
\end{abstract}

